\title{
Graphical Modeling and Animation of Brittle Fracture
}

\author{
James F. O'Brien Jessica K. Hodgins \\ GVU Center and College of Computing \\ Georgia Institute of Technology
}

\begin{abstract}
In this paper, we augment existing techniques for simulating flexible objects to include models for crack initiation and propagation in three-dimensional volumes. By analyzing the stress tensors computed over a finite element model, the simulation determines where cracks should initiate and in what directions they should propagate. We demonstrate our results with animations of breaking bowls, cracking walls, and objects that fracture when they collide. By varying the shape of the objects, the material properties, and the initial conditions of the simulations, we can create strikingly different effects ranging from a wall that shatters when it is hit by a wrecking ball to a bowl that breaks in two when it is dropped on edge.
\end{abstract}

CR Categories: I.3.5 [Computer Graphics]: Computational Geometry and Object Modeling-Physically based modeling; I.3.7 [Computer Graphics]: Three-Dimensional Graphics and Realism-Animation; I.6.8 [Simulation and Modeling]: Types of Simulation-Animation

Keywords: Animation techniques, physically based modeling, simulation, dynamics, fracture, cracking, deformation, finite element method.

\section{Introduction}

With the introduction in 1998 of simulated water in Antz [5, 14] and clothing in Geri's Game [4, 15], passive simulation was clearly demonstrated to be a viable technique for commercial animation. The appeal of using simulation for objects without an internal source of energy is not surprising, as passive objects tend to have many degrees of freedom, making keyframing or motion capture difficult. Furthermore, while passive objects are often essential to the plot of an animation and to the appearance or mood of the piece, they are not characters with their concomitant requirements for control over the subtle details of the motion. Therefore, simulations in which the motion is controlled only by initial conditions, physical equations, and material parameters are often sufficient to produce appealing animations of passive objects.

College of Computing, Georgia Institute of Technology, Atlanta, GA 30332. job@acm.org, jkh@cc.gatech.edu.

Permission to make digital or hard copies of all or part of this work for personal or classroom use is granted without fee provided that copies are not made or distributed for profit or commercial advantage and that copies bear this notice and the full citation on the first page. To copy otherwise, to republish, to post on servers or to redistribute to lists, requires prior specific permission and/or a fee.

SIGGRAPH 99, Los Angeles, CA USA

Copyright ACM 1999 0-201-48560-5/99/08 _ . \$ \$5.00

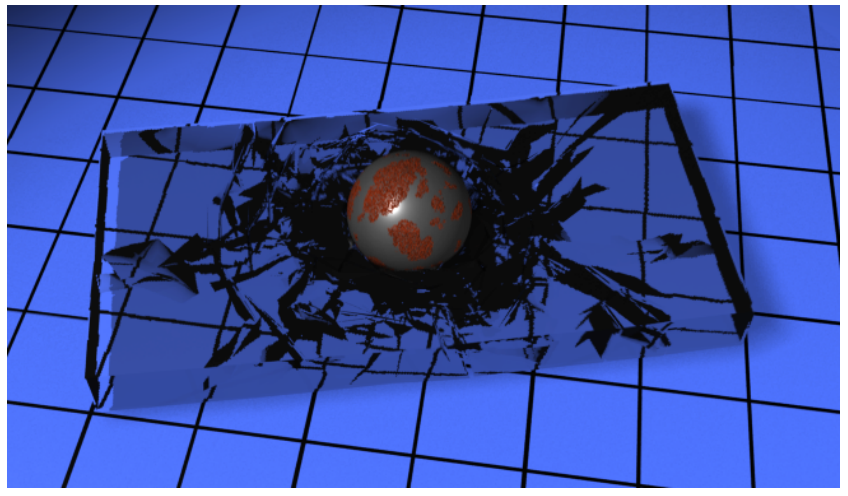

Figure 1: Slab of simulated glass that has been shattered by a heavy weight.

Our approach to animating breaking objects is based on linear elastic fracture mechanics. We model three-dimensional volumes using a finite element method that is based on techniques presented in the computer graphics and mechanical engineering literature $[3,6,18]$. By analyzing the stresses created as a volumetric object deforms, the simulation determines where cracks should begin and in what directions they should propagate. The system accommodates arbitrary propagation directions by dynamically retesselating the mesh. Because cracks are not limited to element boundaries, the models can form irregularly shaped shards and edges as they shatter.

We demonstrate the power of this approach with the following examples: a glass slab that shatters when a weight is dropped onto it (Figure 1), an adobe wall that crumbles under the impact of a wrecking ball (Figure 9), a series of bowls that break when they hit the floor (Figure 11), and objects that break when they collide with each other (Figure 14). To assess the realism of this approach, we compare high-speed video images of a physical bowl dropping onto concrete and a simulated version of the same event (Figure 13).

\section{Background}

In the computer graphics literature, two previous techniques have been developed for modeling dynamic, deformation-induced fracture. In 1988, Terzopoulos and Fleischer [18, 19] presented a general technique for modeling viscoelastic and plastic deformations. Their method used three fundamental metric tensors to define energy functions that measured deformation over curves, surfaces, and volumes. These energy functions provided the basis for a continuous deformation model that they simulated using a variety of discretization methods. One of their methods made use of a finite differencing technique defined by controlled continuity splines [17]. This formulation allowed them to demonstrate how certain fracture effects could be modeled by setting the elastic coefficients between adjacent nodes to zero whenever the distance between the nodes exceeded a threshold. They demonstrated this technique with sheets paper and cloth that could be torn apart. 
In 1991, Norton and his colleagues presented a technique for animating 3D solid objects that broke when subjected to large strains [12]. They simulated a teapot that shattered when dropped onto a table. Their technique used a spring and mass system to model the behavior of the object. When the distance between two attached mass points exceeded a threshold, the simulation severed the spring connection between them. To avoid having flexible strings of partially connected material hanging from the object, their simulation broke an entire cube of springs at once.

Two limitations are inherent in both of these methods. First, when the material fails, the exact location and orientation of the fracture are not known. Rather the failure is defined as the entire connection between two nodes, and the orientation of the fracture plane is left undefined. As a result, these techniques can only realistically model effects that occur on a scale much larger than the inter-node spacing.

The second limitation is that fracture surfaces are restricted to the boundaries in the initial mesh structure. As a result, the fracture pattern exhibits directional artifacts, similar to the "jaggies" that occur when rasterizing a polygonal edge. These artifacts are particularly noticeable when the discretization follows a regular pattern. If an irregular mesh is used, then the artifacts may be partially masked, but the fractures will still be forced onto a path that follows the element boundaries so that the object can break apart only along predefined facets.

Other relevant work in the computer graphics literature includes techniques for modeling static crack patterns and fractures induced by explosions. Hirota and colleagues described how phenomena such as the static crack patterns created by drying mud can be modeled using a mass and spring system attached to an immobile substrate [8]. Mazarak et al. use a voxel-based approach to model solid objects that break apart when they encounter a spherical blast wave [9]. Neff and Fiume use a recursive pattern generator to divide a planar region into polygonal shards that fly apart when acted on by a spherical blast wave [10].

Fracture has been studied more extensively in the mechanics literature, and many techniques have been developed for simulating and analyzing the behavior of materials as they fail. A number of theories may be used to describe when and how a fracture will develop or propagate, and these theories have been employed with various numerical methods including finite element and finite difference methods, boundary integral equations, and molecular particle simulations. A comprehensive review of this work can be found in the book by Anderson [1] and the survey article by Nishioka [11].

Although simulation is used to model fracture both in computer graphics and in engineering, the requirements of the two fields are very different. Engineering applications require that the simulation predict real-world behaviors in an accurate and reliable fashion. In computer animation, what matters is how the fracture looks, how difficult it was to make it look that way, and how long it took. Although the technique presented in this paper was developed using traditional engineering tools, it is an animation technique and relies on a number of simplifications that would be unacceptable in an engineering context.

\section{Deformations}

Fractures arise in materials due to internal stresses created as the material deforms. Our goal is to model these fractures. In order to do so, however, we must first be able to model the deformations that cause them. To provide a suitable framework for modeling fractures, the deformation method must provide information about the magnitude and orientation of the internal stresses, and whether they are tensile or compressive. We would also like to avoid deformation methods in which directional artifacts appear in the stress patterns and propagate to the resulting fracture patterns.
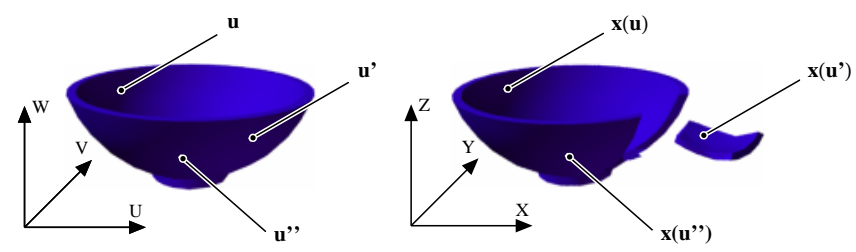

Figure 2: The material coordinates define a 3D parameterization of the object. The function $\boldsymbol{x}(\boldsymbol{u})$ maps points from their location in the material coordinate frame to their location in the world coordinates. A fracture corresponds to a discontinuity in $\boldsymbol{x}(\boldsymbol{u})$.

We derive our deformation technique by defining a set of differential equations that describe the aggregate behavior of the material in a continuous fashion, and then using a finite element method to discretize these equations for computer simulation. This approach is fairly standard, and many different deformation models can be derived in this fashion. The one presented here was designed to be simple, fast, and suitable for fracture modeling.

\subsection{Continuous Model}

Our continuous model is based on continuum mechanics, and an excellent introduction to this area can be found in the text by Fung [7]. The primary assumption in the continuum approach is that the scale of the effects being modeled is significantly greater than the scale of the material's composition. Therefore, the behavior of the molecules, grains, or particles that compose the material can be modeled as a continuous media. Although this assumption is often valid for modeling deformations, macroscopic fractures can be significantly influenced by effects that occur at small scales where this assumption may not be valid. Because we are interested in graphical appearance rather than rigorous physical correctness, we will put this issue aside and assume that a continuum model is adequate.

We begin the description of the continuous model by defining material coordinates that parameterize the volume of space occupied by the object being modeled. Let $\boldsymbol{u}=[u, v, w]^{\top}$ be a vector in $\Re^{3}$ that denotes a location in the material coordinate frame as shown in Figure 2. The deformation of the material is defined by the function $\boldsymbol{x}(\boldsymbol{u})=[x, y, z]^{\top}$ that maps locations in the material coordinate frame to locations in world coordinates. In areas where material exists, $\boldsymbol{x}(\boldsymbol{u})$ is continuous, except across a finite number of surfaces within the volume that correspond to fractures in the material. In areas where there is no material, $\boldsymbol{x}(\boldsymbol{u})$ is undefined.

We make use of Green's strain tensor, $\boldsymbol{\epsilon}$, to measure the local deformation of the material [6]. It can be represented as a $3 \times 3$ symmetric matrix defined by

$$
\epsilon_{i j}=\left(\frac{\partial \boldsymbol{x}}{\partial u_{i}} \cdot \frac{\partial \boldsymbol{x}}{\partial u_{j}}\right)-\delta_{i j}
$$

where $\delta_{i j}$ is the Kronecker delta:

$$
\delta_{i j}=\left\{\begin{array}{lll}
1 & : & i=j \\
0 & : & i \neq j
\end{array}\right.
$$

This strain metric only measures deformation; it is invariant with respect to rigid body transformations applied to $\boldsymbol{x}$ and vanishes when the material is not deformed. It has been used extensively in the engineering literature. Because it is a tensor, its invariants do not depend on the orientation of the material coordinate or world systems. The Euclidean metric tensor used by Terzopoulos and Fleischer [18] differs only by the $\delta_{i j}$ term.

In addition to the strain tensor, we make use of the strain rate tensor, $\boldsymbol{\nu}$, which measures the rate at which the strain is changing. 
It can be derived by taking the time derivative of (1) and is defined by

$$
\nu_{i j}=\left(\frac{\partial \boldsymbol{x}}{\partial u_{i}} \cdot \frac{\partial \dot{\boldsymbol{x}}}{\partial u_{j}}\right)+\left(\frac{\partial \dot{\boldsymbol{x}}}{\partial u_{i}} \cdot \frac{\partial \boldsymbol{x}}{\partial u_{j}}\right)
$$

where an over dot indicates a derivative with respect to time such that $\dot{\boldsymbol{x}}$ is the material velocity expressed in world coordinates.

The strain and strain rate tensors provide the raw information that is required to compute internal elastic and damping forces, but they do not take into account the properties of the material. The stress tensor, $\boldsymbol{\sigma}$, combines the basic information from the strain and strain rate with the material properties and determines forces internal to the material. Like the strain and strain rate tensors, the stress tensor can be represented as a $3 \times 3$ symmetric matrix. It has two components: the elastic stress due to strain, $\boldsymbol{\sigma}^{(\epsilon)}$, and the viscous stress due to strain rate, $\boldsymbol{\sigma}^{(\nu)}$. The total internal stress, is the sum of these two components with

$$
\boldsymbol{\sigma}=\boldsymbol{\sigma}^{(\epsilon)}+\boldsymbol{\sigma}^{(\nu)}
$$

The elastic stress and viscous stress are respectively functions of the strain and strain rate. In their most general linear forms, they are defined as

$$
\begin{aligned}
\sigma_{i j}^{(\epsilon)} & =\sum_{k=1}^{3} \sum_{l=1}^{3} C_{i j k l} \epsilon_{k l} \\
\sigma_{i j}^{(\nu)} & =\sum_{k=1}^{3} \sum_{l=1}^{3} D_{i j k l} \nu_{k l}
\end{aligned}
$$

where $\boldsymbol{C}$ is a set of the 81 elastic coefficients that relate the elements of $\boldsymbol{\epsilon}$ to the elements $\boldsymbol{\sigma}^{(\epsilon)}$, and $\boldsymbol{D}$ is a set of the 81 damping coefficients. $^{1}$

Because both $\boldsymbol{\epsilon}$ and $\boldsymbol{\sigma}^{(\epsilon)}$ are symmetric, many of the coefficients in $\boldsymbol{C}$ are either redundant or constrained, and $\boldsymbol{C}$ can be reduced to 36 independent values that relate the six independent values of $\epsilon$ to the six independent values of $\boldsymbol{\sigma}^{(\epsilon)}$. If we impose the additional constraint that the material is isotropic, then $\boldsymbol{C}$ collapses further to only two independent values, $\mu$ and $\lambda$, which are the Lamé constants of the material. Equation (5) then reduces to

$$
\sigma_{i j}^{(\epsilon)}=\sum_{k=1}^{3} \lambda \epsilon_{k k} \delta_{i j}+2 \mu \epsilon_{i j}
$$

The material's rigidity is determined by the value of $\mu$, and the resistance to changes in volume (dilation) is controlled by $\lambda$.

Similarly, $\boldsymbol{D}$ can be reduced to two independent values, $\phi$ and $\psi$ and (6) then reduces to

$$
\sigma_{i j}^{(\nu)}=\sum_{k=1}^{3} \phi \nu_{k k} \delta_{i j}+2 \psi \nu_{i j} .
$$

The parameters $\mu$ and $\lambda$ will control how quickly the material dissipates internal kinetic energy. Since $\boldsymbol{\sigma}^{(\nu)}$ is derived from the rate at which $\epsilon$ is changing, $\boldsymbol{\sigma}^{(\nu)}$ will not damp motions that are locally rigid, and has the desirable property of dissipating only internal vibrations.

Once we have the strain, strain rate, and stress tensors, we can compute the elastic potential density, $\eta$, and the damping potential density, $\kappa$, at any point in the material using

$$
\eta=\frac{1}{2} \sum_{i=1}^{3} \sum_{j=1}^{3} \sigma_{i j}^{(\epsilon)} \epsilon_{i j},
$$

\footnotetext{
${ }^{1}$ Actually $\boldsymbol{C}$ and $\boldsymbol{D}$ are themselves rank four tensors, and (5) and (6) are normally expressed in this form so that $\boldsymbol{C}$ and $\boldsymbol{D}$ will follow the standard rules for coordinate transforms.
}

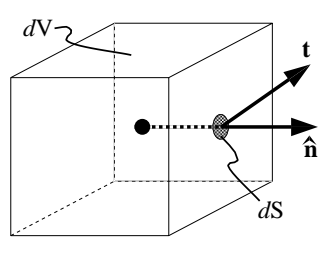

Figure 3: Given a point in the material, the traction, $t$, that acts on the surface element, $d \mathrm{~S}$, of a differential volume, $d \mathrm{~V}$, centered around the point with outward unit normal, $\hat{\boldsymbol{n}}$, is given by $\boldsymbol{t}=\boldsymbol{\sigma} \hat{\boldsymbol{n}}$.

$$
\kappa=\frac{1}{2} \sum_{i=1}^{3} \sum_{j=1}^{3} \sigma_{i j}^{(\nu)} \nu_{i j} .
$$

These quantities can be integrated over the volume of the material to obtain the total elastic and damping potentials. The elastic potential is the internal elastic energy of the material. The damping potential is related to the kinetic energy of the material after subtracting any rigid body motion and normalizing for the material's density.

The stress can also be used to compute the forces acting internal to the material at any location. Let $\hat{\boldsymbol{n}}$ be an outward unit normal direction of a differential volume centered about a point in the material. (See Figure 3.) The traction (force per unit area), $\boldsymbol{t}$, acting on a face perpendicular to the normal is then given by

$$
\boldsymbol{t}=\boldsymbol{\sigma} \hat{\boldsymbol{n}} .
$$

The examples in this paper were generated using this isotropic formulation. However, these techniques do not make use of the strain or strain rate tensors directly; rather they rely only on the stress. Switching to the anisotropic formulation, or even to a nonlinear stress to strain relation, would not require any significant changes.

\subsection{Finite Element Discretization}

Before we can model a material's behavior using this continuous model, it must be discretized in a way that is suitable for computer simulation. Two commonly used techniques are the finite difference and finite element methods.

A finite difference method divides the domain of the material into a regular lattice and then uses numerical differencing to approximate the spatial derivatives required to compute the strain and strain rate tensors. This approach is well suited for problems with a regular structure but becomes complicated when the structure is irregular.

A finite element method partitions the domain of the material into distinct sub-domains, or elements as shown in Figure 4. Within each element, the material is described locally by a function with some finite number of parameters. The function is decomposed into a set of orthogonal shape, or basis, functions that are each associated with one of the nodes on the boundary of the element. Adjacent elements will have nodes in common, so that the mesh defines a piecewise function over the entire material domain.

Our discretization employs tetrahedral finite elements with linear polynomial shape functions. By using a finite element method, the mesh can be locally aligned with the fracture surfaces, thus avoiding the previously mentioned artifacts. Just as triangles can be used to approximate any surface, tetrahedra can be used to approximate arbitrary volumes. Additionally, when tetrahedra are split along a fracture plane, the resulting pieces can be decomposed exactly into more tetrahedra.

We chose to use linear elements because higher-order elements are not cost effective for modeling fracture boundaries. Although higher-order polynomials provide individual elements with many 


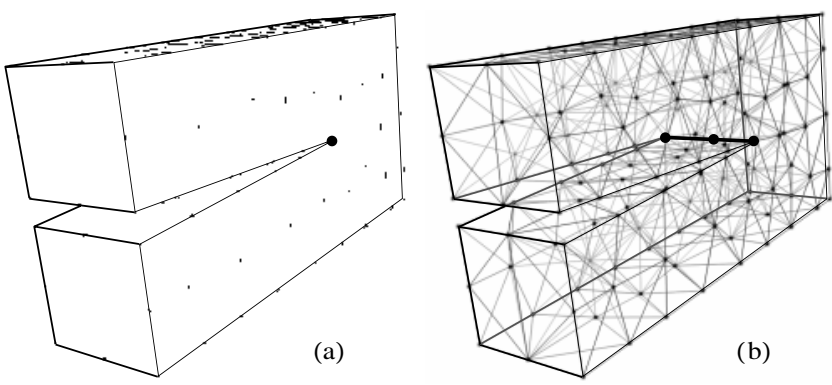

Figure 4: Tetrahedral mesh for a simple object. In (a), only the external faces of the tetrahedra are drawn; in (b) the internal structure is shown.

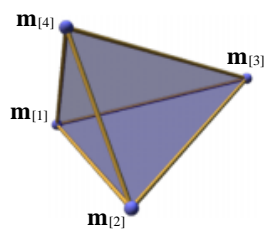

(a)

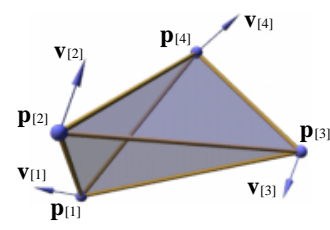

(b)
Figure 5: A tetrahedral element is defined by its four nodes. Each node has (a) a location in the material coordinate system and (b) a position and velocity in the world coordinate system.

degrees of freedom for deformation, they have few degrees of freedom for modeling fracture because the shape of a fracture is defined as a boundary in material coordinates. In contrast, with linear tetrahedra, each degree of freedom in the world space corresponds to a degree of freedom in the material coordinates. Furthermore, whenever an element is created, its basis functions must be computed. For high-degree polynomials, this computation is relatively expensive. For systems where the mesh is constant, the cost is amortized over the course of the simulation. However, as fractures develop and parts of the object are remeshed, the computation of basis matrices can become significant.

Each tetrahedral element is defined by four nodes. A node has a position in the material coordinates, $\boldsymbol{m}$, a position in the world coordinates, $\boldsymbol{p}$, and a velocity in world coordinates, $\boldsymbol{v}$. We will refer to the nodes of a given element by indexing with square brackets. For example, $\boldsymbol{m}_{[2]}$ is the position in material coordinates of the element's second node. (See Figure 5.)

Barycentric coordinates provide a natural way to define the linear shape functions within an element. Let $\boldsymbol{b}=\left[b_{1}, b_{2}, b_{3}, b_{4}\right]^{\top}$ be barycentric coordinates defined in terms of the element's material coordinates so that

$$
\left[\begin{array}{l}
\boldsymbol{u} \\
1
\end{array}\right]=\left[\begin{array}{cccc}
\boldsymbol{m}_{[1]} & \boldsymbol{m}_{[2]} & \boldsymbol{m}_{[3]} & \boldsymbol{m}_{[4]} \\
1 & 1 & 1 & 1
\end{array}\right] \boldsymbol{b} .
$$

These barycentric coordinates may also be used to interpolate the node's world position and velocity with

$$
\begin{aligned}
& {\left[\begin{array}{c}
\boldsymbol{x} \\
1
\end{array}\right]=\left[\begin{array}{cccc}
\boldsymbol{p}_{[1]} & \boldsymbol{p}_{[2]} & \boldsymbol{p}_{[3]} & \boldsymbol{p}_{[4]} \\
1 & 1 & 1 & 1
\end{array}\right] \boldsymbol{b}} \\
& {\left[\begin{array}{c}
\dot{\boldsymbol{x}} \\
1
\end{array}\right]=\left[\begin{array}{cccc}
\boldsymbol{v}_{[1]} & \boldsymbol{v}_{[2]} & \boldsymbol{v}_{[3]} & \boldsymbol{v}_{[4]} \\
1 & 1 & 1 & 1
\end{array}\right] \boldsymbol{b} \text {. }}
\end{aligned}
$$

To determine the barycentric coordinates of a point within the element specified by its material coordinates, we invert (12) and obtain

$$
\boldsymbol{b}=\boldsymbol{\beta}\left[\begin{array}{l}
\boldsymbol{u} \\
1
\end{array}\right]
$$

where $\boldsymbol{\beta}$ is defined by

$$
\boldsymbol{\beta}=\left[\begin{array}{cccc}
\boldsymbol{m}_{[1]} & \boldsymbol{m}_{[2]} & \boldsymbol{m}_{[3]} & \boldsymbol{m}_{[4]} \\
1 & 1 & 1 & 1
\end{array}\right]^{-1} .
$$

Combining (15) with (13) and (14) yields functions that interpolate the world position and velocity within the element in terms of the material coordinates:

$$
\begin{aligned}
& \boldsymbol{x}(\boldsymbol{u})=\boldsymbol{P} \boldsymbol{\beta}\left[\begin{array}{l}
\boldsymbol{u} \\
1
\end{array}\right] \\
& \dot{\boldsymbol{x}}(\boldsymbol{u})=\boldsymbol{V} \boldsymbol{\beta}\left[\begin{array}{l}
\boldsymbol{u} \\
1
\end{array}\right]
\end{aligned}
$$

where $\boldsymbol{P}$ and $\boldsymbol{V}$ are defined as

$$
\begin{aligned}
\boldsymbol{P} & =\left[\boldsymbol{p}_{[1]} \boldsymbol{p}_{[2]} \boldsymbol{p}_{[3]} \boldsymbol{p}_{[4]}\right] \\
\boldsymbol{V} & =\left[\boldsymbol{v}_{[1]} \boldsymbol{v}_{[2]} \boldsymbol{v}_{[3]} \boldsymbol{v}_{[4]}\right] .
\end{aligned}
$$

Note that the rows of $\boldsymbol{\beta}$ are the coefficients of the shape functions, and $\boldsymbol{\beta}$ needs to be computed only when an element is created or the material coordinates of its nodes change. For non-degenerate elements, the matrix in (16) is guaranteed to be non-singular, however elements that are nearly co-planar will cause $\boldsymbol{\beta}$ to be ill-conditioned and adversely affect the numerical stability of the system.

Computing the values of $\epsilon$ and $\boldsymbol{\nu}$ within the element requires the first partials of $\boldsymbol{x}$ with respect to $\boldsymbol{u}$ :

$$
\begin{aligned}
\frac{\partial \boldsymbol{x}}{\partial u_{i}} & =\boldsymbol{P} \boldsymbol{\beta} \boldsymbol{\delta}_{i} \\
\frac{\partial \dot{\boldsymbol{x}}}{\partial u_{i}} & =\boldsymbol{V} \boldsymbol{\beta} \boldsymbol{\delta}_{i}
\end{aligned}
$$

where

$$
\boldsymbol{\delta}_{i}=\left[\begin{array}{llll}
\delta_{i 1} & \delta_{i 2} & \delta_{i 3} & 0
\end{array}\right]^{\top} .
$$

Because the element's shape functions are linear, these partials are constant within the element.

The element will exert elastic and damping forces on its nodes. The elastic force on the $i$ th node, $\boldsymbol{f}_{[i]}^{(\epsilon)}$, is defined as the partial of the elastic potential density, $\eta$, with respect to $\boldsymbol{p}_{[i]}$ integrated over the volume of the element. Given $\boldsymbol{\sigma}^{(\epsilon)}, \boldsymbol{\beta}$, and the positions in world space of the four nodes we can compute the elastic force by

$$
\boldsymbol{f}_{[i]}^{(\epsilon)}=\frac{\operatorname{vol}}{2} \sum_{j=1}^{4} \boldsymbol{p}_{[j]} \sum_{k=1}^{3} \sum_{l=1}^{3} \beta_{j l} \beta_{i k} \sigma_{k l}^{(\epsilon)}
$$

where

$$
\operatorname{vol}=\frac{1}{6}\left[\left(\boldsymbol{m}_{[2]}-\boldsymbol{m}_{[1]}\right) \times\left(\boldsymbol{m}_{[3]}-\boldsymbol{m}_{[1]}\right)\right] \cdot\left(\boldsymbol{m}_{[4]}-\boldsymbol{m}_{[1]}\right) .
$$

Similarly, the damping force on the $i$ th node, $\boldsymbol{f}_{[i]}^{(\nu)}$, is defined as the partial of the damping potential density, $\kappa$, with respect to $\boldsymbol{v}_{[i]}$ integrated over the volume of the element. This quantity can be computed with

$$
\boldsymbol{f}_{[i]}^{(\nu)}=\frac{\operatorname{vol}}{2} \sum_{j=1}^{4} \boldsymbol{p}_{[j]} \sum_{k=1}^{3} \sum_{l=1}^{3} \beta_{j l} \beta_{i k} \sigma_{k l}^{(\nu)} .
$$

Summing these two forces, the total internal force that an element exerts on a node is

$$
\boldsymbol{f}_{[i]}^{\mathrm{el}}=\frac{\operatorname{vol}}{2} \sum_{j=1}^{4} \boldsymbol{p}_{[j]} \sum_{k=1}^{3} \sum_{l=1}^{3} \beta_{j l} \beta_{i k} \sigma_{k l},
$$


and the total internal force acting on the node is obtained by summing the forces exerted by all elements that are attached to the node.

As the element is compressed to less than about $30 \%$ of its material volume, the gradient of $\eta$ and $\kappa$ start to vanish causing the resisting forces to fall off. We have not found this to be a problem as even the more squishy of the materials that we have modeled conserve their volume to within a few percent.

Using a lumped mass formulation, the mass contributed by an element to each one of its nodes is determined by integrating the material density, $\rho$, over the element shape function associated with that node. In the case of tetrahedral elements with linear shape functions, this mass contribution is simply $\rho \mathrm{vol} / 4$.

The derivations above are sufficient for a simulation that uses an explicit integration scheme. Additional work, including computing the Jacobian of the internal forces, is necessary for implicit integration scheme. (See for example [2] and [3].)

\subsection{Collisions}

In addition to the forces internal to the material, the system computes collision forces. The collision forces are computed using a penalty method that is applied when two elements intersect or if an element violates another constraint such as the ground. Although penalty methods are often criticized for creating stiff equations, we have found that for the materials we are modeling the internal forces are at least as stiff as the penalty forces. Penalty forces have the advantage of being very fast to compute. We have experimented with two different penalty criteria: node penetration and overlap volume. The examples presented in this paper were computed with the node penetration criteria; additional examples on the conference proceedings CD-ROM were computed with the overlap volume criteria.

The node penetration criteria sets the penalty force to be proportional to the distance that a node has penetrated into another element. The penalty force acts in the direction normal to the penetrated surface. The reaction force is distributed over the penetrated element's nodes so that the net force and moment are the negation of the penalty force and moment acting at the penetrating node. This test will not catch all collisions, and undetected intersecting tetrahedra may become locked together. It is however, fast to compute, easy to implement, and adequate for situations that do not involve complex collision interactions.

The overlap volume criteria is more robust than the node penetration method, but it is also slower to compute and more complex to implement. The intersection of two tetrahedral elements is computed by clipping the faces of each tetrahedron against the other. The resulting polyhedron is then used to compute the volume and center of mass of the intersecting region. The area weighted normals of the faces of the polyhedron that are contributed by one of the tetrahedra are summed to compute the direction that the penalty force acts in. A similar computation can be performed for the other tetrahedra, or equivalently the direction can be negated. Provided that neither tetrahedra is completely contained within the other, this criteria is more robust than the node penetration criteria. Additionally, the forces computed with this method do not depend on the object tessellation.

Computing the intersections within the mesh can be very expensive, and we use a bounding hierarchy scheme with cached traversals to help reduce this cost.

\section{Fracture Modeling}

Our fracture model is based on the theory of linear elastic fracture mechanics [1]. The primary distinction between this and other the-

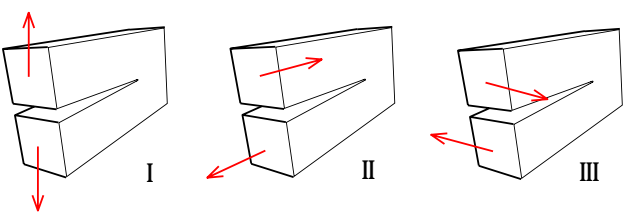

Figure 6: Three loading modes that can be experienced by a crack. Mode I: Opening, Mode II: In-Plane Shear, and Mode III: Out-ofPlane Shear. Adapted from Anderson [1].

ories of fracture is that the region of plasticity near the crack tip ${ }^{2}$ is neglected. Because we are not modeling the energy dissipated by this plastic region, modeled materials will be brittle. This statement does not mean that they are weak; rather the term "brittle" refers to the fact that once the material has begun to fail, the fractures will have a strong tendency to propagate across the material as they are driven by the internally stored elastic energy.

There are three loading modes by which forces can be applied to a crack causing it to open further. (See Figure 6.) In most circumstances, some combination of these modes will be active, producing a mixed mode load at the crack tip. For all three cases, as well as mixed mode situations, the behavior of the crack can be resolved by analyzing the forces acting at the crack tip: tensile forces that are opposed by other tensile forces will cause the crack to continue in a direction that is perpendicular to the direction of largest tensile load, and conversely, compressive loads will tend to arrest a crack to which they are perpendicular.

The finite element model describes the surface of a fracture with elements that are adjacent in material coordinates but that do not share nodes across the internal surface. The curve that represents the crack tip is then implicitly defined in a piecewise linear fashion by the nodes that border the fracture surface, and further extension of the crack may be determined by analyzing the internal forces acting on these nodes.

We will also use the element nodes to determine where a crack should be initiated. While this strategy could potentially introduce unpleasant artifacts, we note that because the surface of an object is defined by a polygonal boundary (the outer faces of the tetrahedra) there will always be a node located at any concavities. Because concavities are precisely the locations where cracks commonly begin, we believe that this decision is acceptable.

Our fracture algorithm is as follows: after every time step, the system resolves the internal forces acting on all nodes into their tensile and compressive components, discarding any unbalanced portions. At each node, the resulting forces are then used to form a tensor that describes how the internal forces are acting to separate that node. If the action is sufficiently large, the node is split into two distinct nodes and a fracture plane is computed. All elements attached to the node are divided along the plane with the resulting tetrahedra assigned to one or the other incarnations of the split node, thus creating a discontinuity in the material. Any cached values, such as the node mass or the element shape functions, are recomputed for the affected elements and nodes. With this technique, the location of a fracture or crack tip need not be explicitly recorded unless this information is useful for some other purpose, such as rendering.

\footnotetext{
${ }^{2}$ The term "crack tip" implies that the fracture will have a single point at its tip. In general, the front of the crack will not be a single point; rather it will be a curve that defines the boundary of the surface discontinuity in material coordinates. (See Figure 4.) Nevertheless, we will refer to this front as the crack tip.
} 


\subsection{Force Decomposition}

The forces acting on a node are decomposed by first separating the element stress tensors into tensile and compressive components. For a given element in the mesh, let $v^{i}(\boldsymbol{\sigma})$, with $i \in\{1,2,3\}$, be the $i$ th eigenvalue of $\boldsymbol{\sigma}$, and let $\hat{\mathbf{n}}^{i}(\boldsymbol{\sigma})$ be the corresponding unit length eigenvector. Positive eigenvalues correspond to tensile stresses and negative ones to compressive stresses. Since $\boldsymbol{\sigma}$ is real and symmetric, it will have three real, not necessarily unique, eigenvalues. In the case where an eigenvalue has multiplicity greater than one, the eigenvectors are selected arbitrarily to orthogonally span the appropriate subspace [13].

Given a vector $\boldsymbol{a}$ in $\Re^{3}$, we can construct a $3 \times 3$ symmetric matrix, $\mathbf{m}(\boldsymbol{a})$ that has $|\boldsymbol{a}|$ as an eigenvalue with $\boldsymbol{a}$ as the corresponding eigenvector, and with the other two eigenvalues equal to zero. This matrix is defined by

$$
\mathbf{m}(\boldsymbol{a})=\left\{\begin{array}{cl}
\boldsymbol{a} \boldsymbol{a}^{\top} /|\boldsymbol{a}| & : \quad \boldsymbol{a} \neq \mathbf{0} \\
\mathbf{0} & : \quad \boldsymbol{a}=\mathbf{0}
\end{array}\right.
$$

The tensile component, $\boldsymbol{\sigma}^{+}$, and compressive component, $\boldsymbol{\sigma}^{-}$, of the stress within the element can now be computed by

$$
\begin{aligned}
& \boldsymbol{\sigma}^{+}=\sum_{i=1}^{3} \max \left(0, v^{i}(\boldsymbol{\sigma})\right) \mathbf{m}\left(\hat{\mathbf{n}}^{i}(\boldsymbol{\sigma})\right) \\
& \boldsymbol{\sigma}^{-}=\sum_{i=1}^{3} \min \left(0, v^{i}(\boldsymbol{\sigma})\right) \mathbf{m}\left(\hat{\mathbf{n}}^{i}(\boldsymbol{\sigma})\right) .
\end{aligned}
$$

Using this decomposition, the force that an element exerts on a node can be separated into a tensile component, $\boldsymbol{f}_{[i]}^{+}$, and a compressive component, $\boldsymbol{f}_{[i]}^{-}$. This separation is done by reevaluating the internal forces exerted on the nodes using (27) with $\boldsymbol{\sigma}^{+}$or $\boldsymbol{\sigma}^{-}$ substituted for $\boldsymbol{\sigma}$. Thus the tensile component is

$$
\boldsymbol{f}_{[i]}^{+}=\frac{\operatorname{vol}}{2} \sum_{j=1}^{4} \boldsymbol{p}_{[j]} \sum_{k=1}^{3} \sum_{l=1}^{3} \beta_{j l} \beta_{i k} \sigma_{k l}^{+} .
$$

The compressive component can be computed similarly, but because $\boldsymbol{\sigma}=\boldsymbol{\sigma}^{+}+\boldsymbol{\sigma}^{-}$, it can be computed more efficiently using $\boldsymbol{f}_{[i]}=\boldsymbol{f}_{[i]}^{+}+\boldsymbol{f}_{[i]}^{-}$.

Each node will now have a set of tensile and a set of compressive forces that are exerted by the elements attached to it. For a given node, we denote these sets as $\left\{\boldsymbol{f}^{+}\right\}$and $\left\{\boldsymbol{f}^{-}\right\}$respectively. The unbalanced tensile load, $\boldsymbol{f}^{+}$is simply the sum over $\left\{\boldsymbol{f}^{+}\right\}$, and the unbalanced compressive load, $\boldsymbol{f}^{-}$, is the sum over $\left\{\boldsymbol{f}^{-}\right\}$.

\subsection{The Separation Tensor}

We describe the forces acting at the nodes using a stress variant that we call the separation tensor, $\varsigma$. The separation tensor is formed from the balanced tensile and compressive forces acting at each node and is computed by

$$
\boldsymbol{\varsigma}=\frac{1}{2}\left(-\mathbf{m}\left(\boldsymbol{f}^{+}\right)+\sum_{\boldsymbol{f} \in\left\{\boldsymbol{f}^{+}\right\}} \mathbf{m}(\boldsymbol{f})+\mathbf{m}\left(\boldsymbol{f}^{-}\right)-\sum_{\boldsymbol{f} \in\left\{\boldsymbol{f}^{-}\right\}} \mathbf{m}(\boldsymbol{f})\right) .
$$

It does not respond to unbalanced actions that would produce a rigid translation, and is invariant with respect to transformations of both the material and world coordinate systems.

The separation tensor is used directly to determine whether a fracture should occur at a node. Let $\mathrm{v}^{+}$be the largest positive eigenvalue of $\boldsymbol{\varsigma}$. If $\mathrm{v}^{+}$is greater than the material toughness, $\tau$, then the
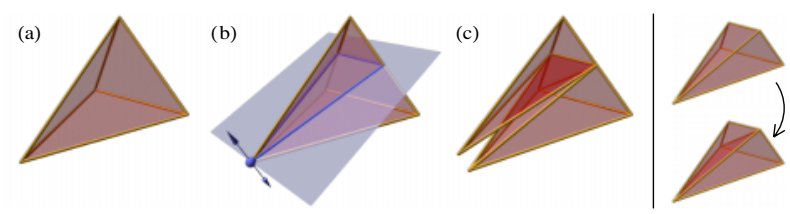

Figure 7: Diagram showing how an element is split by the fracture plane. (a) The initial tetrahedral element. (b) The splitting node and fracture plane are shown in blue. (c) The element is split along the fracture plane into two polyhedra that are then decomposed into tetrahedra. Note that the two nodes created from the splitting node are co-located, the geometric displacement shown in (c) only illustrates the location of the fracture discontinuity.
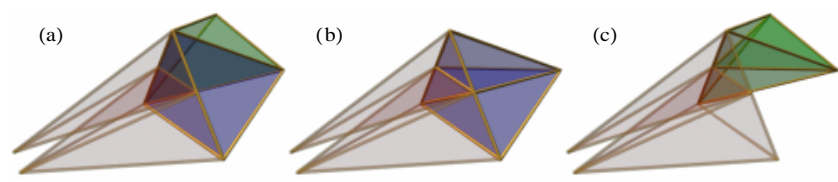

Figure 8: Elements that are adjacent to an element that has been split by a fracture plane must also be split to maintain mesh consistency. (a) Neighboring tetrahedra prior to split. (b) Face neighbor after split. (c) Edge neighbor after split.

material will fail at the node. The orientation in world coordinates of the fracture plane is perpendicular to $\hat{\mathbf{n}}^{+}$, the eigenvalue of $\varsigma$ that corresponds to $\mathrm{v}^{+}$. In the case where multiple eigenvalues are greater than $\tau$, multiple fracture planes may be generated by first generating the plane for the largest value, remeshing (see below), and then recomputing the new value for $\varsigma$ and proceeding as above.

\subsection{Local Remeshing}

Once the simulation has determined the location and orientation of a new fracture plane, the mesh must be modified to reflect the new discontinuity. It is important that the orientation of the fracture be preserved, as approximating it with the existing element boundaries would create undesirable artifacts. To avoid this potential difficulty, the algorithm remeshes the local area surrounding the new fracture by splitting elements that intersect the fracture plane and modifying neighboring elements to ensure that the mesh stays self-consistent.

First, the node where the fracture originates is replicated so that there are now two nodes, $n^{+}$and $n^{-}$with the same material position, world position, and velocity. The masses will be recalculated later. The discontinuity passes "between" the two co-located nodes. The positive side of the fracture plane is associated with $n^{+}$and the negative side with $n^{-}$.

Next, all elements that were attached to the original node are examined, comparing the world location of their nodes to the fracture plane. If an element is not intersected by the fracture plane, then it is reassigned to either $n^{+}$or $n^{-}$depending on which side of the plane it lies.

If the element is intersected by the fracture plane, it is split along the plane. (See Figure 7.) A new node is created along each edge that intersects the plane. Because all elements must be tetrahedra, in general each intersected element will be split into three tetrahedra. One of the tetrahedra will be assigned to one side of the plane and the other two to the other side. Because the two tetrahedra that are on the same side of the plane both share either $n^{+}$or $n^{-}$, the discontinuity does not pass between them.

In addition to the elements that were attached to the original node, it may be necessary to split other elements so that the mesh 

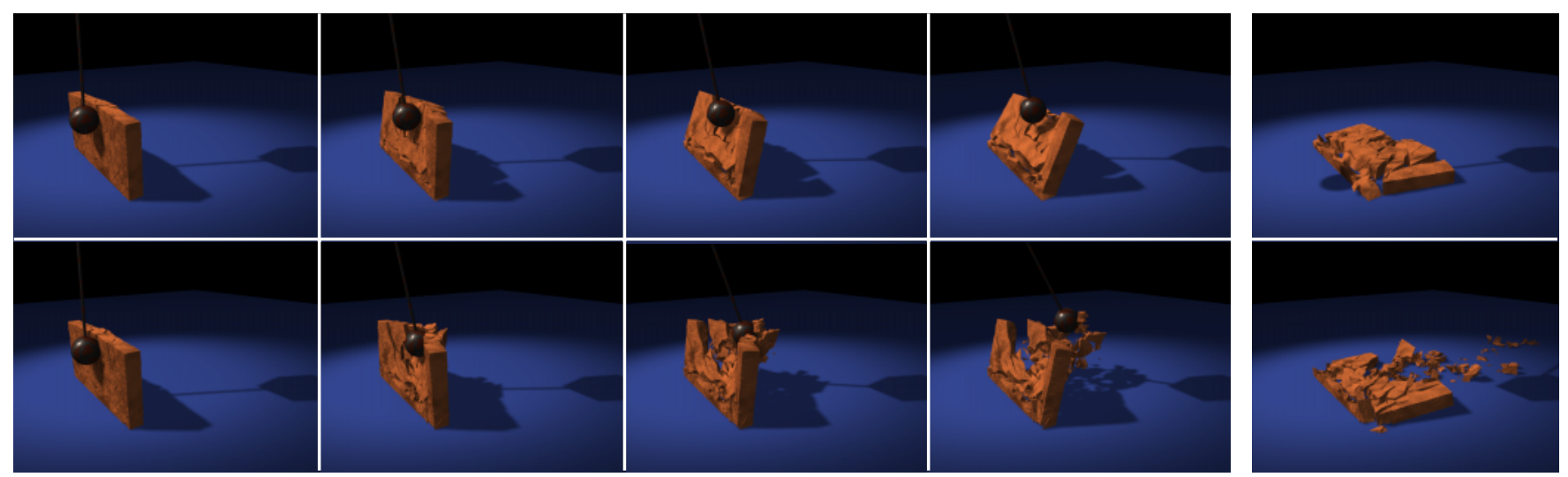

Figure 9: Two adobe walls that are struck by wrecking balls. Both walls are attached to the ground. The ball in the second row has $50 \times$ the mass of the first. Images are spaced apart $133.3 \mathrm{~ms}$ in the first row and $66.6 \mathrm{~ms}$ in the second. The rightmost images show the final configurations.

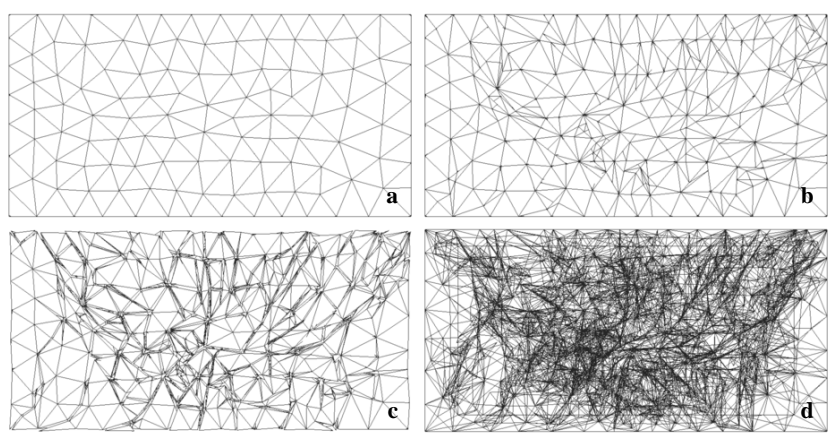

Figure 10: Mesh for adobe wall. (a) The facing surface of the initial mesh used to generate the wall shown in Figure 9. (b) The mesh after being struck by the wrecking ball, reassembled. (c) Same as (b), with the cracks emphasized. (d) Internal fractures shown as wireframe.

stays consistent. In particular, an element must be split if the face or edge between it and another element that was attached to the original node has been split. (See Figure 8.) To prevent the remeshing from cascading across the entire mesh, these splits are done so that the new tetrahedra use only the original nodes and the nodes created by the intersection splits. Because no new nodes are created, the effect of the local remeshing is limited to the elements that are attached to the node where the fracture originated and their immediate neighbors. Because the tetrahedra formed by the secondary splits do not attach to either $n^{+}$or $n^{-}$, the discontinuity does not pass between them.

Finally, after the local remeshing has been completed, any cached values that have become invalid must be recomputed. In our implementation, these values include the element basis matrix, $\boldsymbol{\beta}$, and the node masses.

Two additional subtleties must also be considered. The first subtlety occurs when an intersection split involves an edge that is formed only by tetrahedra attached to the node where the crack originated. When this happens, the fracture has reached a boundary in the material, and the discontinuity should pass through the edge. Remeshing occurs as above, except that two nodes are created on the edge and one is assigned to each side of the discontinuity.

Second, the fracture plane may pass arbitrarily close to an existing node producing arbitrarily ill-conditioned tetrahedra. To avoid this, we employ two thresholds, one the distance between the frac- ture plane and an existing node, and the other on the angle between the fracture plane and a line from the node where the split originated to the existing node. If either of these thresholds are not met, then the intersection split is snapped to the existing node. In our results, we have used thresholds of $5 \mathrm{~mm}$ and 0.1 radians.

\section{Results and Discussion}

To demonstrate some of the effects that can be generated with this fracture technique, we have animated a number of scenes that involve objects breaking. Figure 1 shows a plate of glass that has had a heavy weight dropped on it. The area in the immediate vicinity of the impact has been crushed into many small fragments. Further away from the weight, a pattern of radial cracks has developed.

Figure 9 shows two walls being struck by wrecking balls. In the first sequence, the wall develops a network of cracks as it absorbs most of the ball's energy during the initial impact. In the second sequence, the ball's mass is $50 \times$ greater, and the wall shatters when it is struck. The mesh used to generate the wall sequences is shown in Figure 10. The initial mesh contains only 338 nodes and 1109 elements. By the end of the sequence, the mesh has grown to 6892 nodes and 8275 elements. These additional nodes and elements are created where fractures occur; a uniform mesh would require many times this number of nodes and elements to achieve a similar result.

Figure 11 shows the final frames from four animations of bowls that were dropped onto a hard surface. Other than the toughness, $\tau$, of the material, the four simulations are identical. The first bowl develops only a few cracks; the weakest breaks into many pieces.

Because this system works with solid tetrahedral volumes rather than with the polygonal boundary representations created by most modeling packages, boundary models must be converted before they can be used. A number of systems are available for creating tetrahedral meshes from polygonal boundaries. The models that we used in these examples were generated either from a CSG description or a polygonal boundary representation using NETGEN, a publicly available mesh generation package [16].

Although our approach avoids the "jaggy" artifacts in the fracture patterns caused by the underlying mesh, there remain ways in which the results of a simulation are influenced by the mesh structure. The most obvious is that the deformation of the material is limited by the degrees of freedom in the mesh, which in turn limits how the material can fracture. This limitation will occur with any discrete system. The technique also limits where a fracture may ini- 

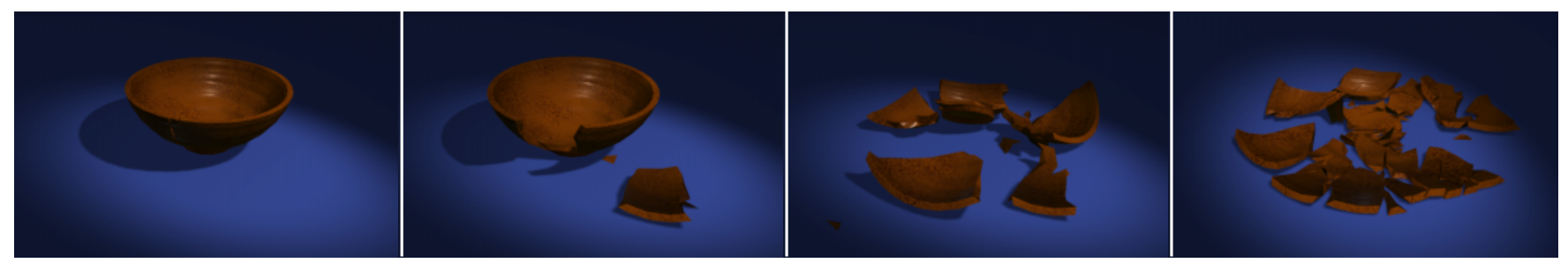

Figure 11: Bowls with successively lower toughness values, $\tau$. Each of the bowls were dropped from the same height. Other than $\tau$, the bowls have same material properties.

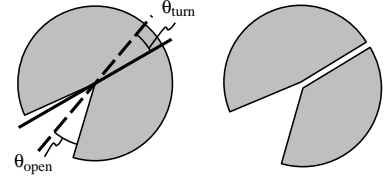

(a)
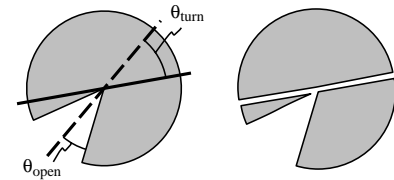

(b)

Figure 12: Back-cracking during fracture advance. The dashed line is the axis of the existing crack. Cracks advance by splitting elements along a fracture plane, shown as a solid line, computed from the separation tensor. (a) If the crack does not turn sharply, then only elements in front of the tip will be split. (b) If the crack turns at too sharp an angle, then the backwards direction may not fall inside of the existing failure and a spurious bifurcation will occur.

tiate by examining only the existing nodes. This assumption means that very coarse mesh sizes might behave in an unintuitive fashion. However, nodes correspond to the locations where a fracture is most likely to begin; therefore, with a reasonable grid size, this limitation is not a serious handicap.

A more serious limitation is related to the speed at which a crack propagates. Currently, the distance that a fracture may travel during a time step is determined by the size of the existing mesh elements. The crack may either split an element or not; it cannot travel only a fraction of the distance across an element. If a crack were being opened slowly by an applied load on a model with a coarse resolution mesh, this limitation would lead to a "button popping" effect where the crack would travel across one element, pause until the stress built up again, and then move across the next element. A second type of artifact may occur if the crack's speed should be significantly greater than the element width divided by the simulation time step. In this case, a high stress area will race ahead of the crack tip, causing spontaneous failures to occur in the material. Although we have not observed these phenomena in our examples, developing an algorithm that allows a fracture to propagate arbitrary distances is an area for future work.

Another limitation stems from the fact that while the fracture plane's orientation is well defined, the crack tip's forward direction is not. As shown in Figure 12, if the cracks turns at an angle greater than half the angle at the crack tip, then a secondary fracture will develop in the opposite direction to the crack's advance. While this effect is likely present in some of our examples, it does not appear to have a significant impact on the quality of the results. If the artifacts were to be a problem, they could be suppressed by explicitly tracking the fracture propagation directions within the mesh.

The simulation parameters used to generate the examples in this paper are listed in Table 1 along with the computation time required to generate one second of animation. While the material density values, $\rho$, are appropriate for glass, stone, or ceramic, we used values for the Lamé constants, $\lambda$ and $\mu$, that are significantly less than those of real materials. Larger values would make the simulated materials appear stiffer, but would also require smaller time steps.

The values that we have selected represent a compromise between realistic behavior and reasonable computation time.

Our current implementation can switch between either a forward Euler integration scheme or a second order Taylor integrator. Both of these techniques are explicit integration schemes, and subject to stability limits that require very small time steps for stiff materials. Although semi-implicit integration methods have error bounds similar to those of explicit methods, the semi-implicit integrators tend to drive errors towards zero rather than infinity so that they are stable at much larger time steps. Other researchers have shown that by taking advantage of this property, a semi-implicit integrator can be used to realize speed ups of two or three orders of magnitude when modeling object deformation [2]. Unfortunately, it may be difficult to realize these same improvements when fracture propagation is part of the simulation. As discussed above, the crack speed is limited in inverse proportion to the time step size, and the large time steps that might be afforded by a semi-implicit integrator could cause spontaneous material failure to proceed crack advance. We are currently investigating how our methods may be modified to be compatible with large time step integration schemes.

Many materials and objects in the real world are not homogeneous, and it would be interesting to develop graphical models for animating them as they fail. For example, a brick wall is made up of mortar and bricks arranged in a regular fashion, and if simulated in a situation like our wall example, a distinct pattern would be created. Similarly, the connection between a handmade cup and its handle is often weak because of the way in which the handle is attached.

One way to assess the realism of an animation technique is by comparing it with the real world. Figure 13 shows high-speed video footage of a physical bowl as it falls onto its edge compared to our imitation of the real-world scene. Although the two sets of fracture patterns are clearly different, the simulated bowl has some qualitative similarities to the real one. Both initially fail along the leading edge where they strike the ground, and subsequently develop vertical cracks before breaking into several large pieces.

\section{Acknowledgments}

The authors would like to thank Wayne L. Wooten of Pixar Animation Studios for lighting, shading, and rendering the images for many of the figures in this paper. We would also like to thank Ari Glezer and Bojan Vukasinovic of the School Mechanical Engineering at the Georgia Institute of Technology for their assistance and the use of the high-speed video equipment. Finally, we would like to thank those in the Animation Lab who lent a hand to ensure that we made the submission deadline.

This project was supported in part by NSF NYI Grant No. IRI9457621, Mitsubishi Electric Research Laboratory, and a Packard Fellowship. The first author was supported by a Fellowship from the Intel Foundation. 


\begin{tabular}{|c|c|c|c|c|c|c|c|c|c|c|}
\hline \multirow[b]{2}{*}{ Example } & \multirow[b]{2}{*}{ Figure } & \multirow[b]{2}{*}{$\lambda\left(N / m^{2}\right)$} & \multirow[b]{2}{*}{$\mu\left(N / m^{2}\right)$} & \multicolumn{2}{|c|}{ Material Parameters } & \multirow[b]{2}{*}{$\rho\left(k g / m^{3}\right)$} & \multirow[b]{2}{*}{$\tau\left(N / m^{2}\right)$} & \multicolumn{3}{|c|}{$\begin{array}{c}\text { Minutes of Computation } \\
\text { Time per Simulation Second }\end{array}$} \\
\hline & & & & $\phi\left(N s / m^{2}\right)$ & $\psi\left(N s / m^{2}\right)$ & & & Minimum & Maximum & Average \\
\hline Glass & 1 & $1.04 \times 10^{8}$ & $1.04 \times 10^{8}$ & 0 & 6760 & 2588 & 10140 & 75 & 667 & 273 \\
\hline Wall \#1 & $9 . \mathrm{a}$ & $6.03 \times 10^{8}$ & $1.21 \times 10^{8}$ & 3015 & 6030 & 2309 & 6030 & 75 & 562 & 399 \\
\hline Wall \#2 & $9 . \mathrm{b}$ & 0 & $1.81 \times 10^{8}$ & 0 & 18090 & 2309 & 6030 & 75 & 2317 & 1098 \\
\hline Bowl \#1 & 11.a & $2.65 \times 10^{6}$ & $3.97 \times 10^{6}$ & 264 & 397 & 1013 & 52.9 & 90 & 120 & 109 \\
\hline Bowl \#2 & 11.b & $2.65 \times 10^{6}$ & $3.97 \times 10^{6}$ & 264 & 397 & 1013 & 39.6 & 82 & 135 & 115 \\
\hline Bowl \#3 & 11.c & $2.65 \times 10^{6}$ & $3.97 \times 10^{6}$ & 264 & 397 & 1013 & 33.1 & 90 & 150 & 127 \\
\hline Bowl \#4 & 11.d & $2.65 \times 10^{6}$ & $3.97 \times 10^{6}$ & 264 & 397 & 1013 & 13.2 & 82 & 187 & 156 \\
\hline Comp. Bowl & 13 & 0 & $5.29 \times 10^{7}$ & 0 & 198 & 1013 & 106 & 247 & 390 & 347 \\
\hline The End & 14 & 0 & $9.21 \times 10^{6}$ & 0 & 9.2 & 705 & 73.6 & 622 & 6667 & 4665 \\
\hline
\end{tabular}

Table 1: Material parameters and simulation times for examples. The times listed reflect the total number of minutes required to compute one second of simulated data, including graphics and file I/O. Times were measured on an SGI O2 with a 195 MHz MIPS R10K processor.
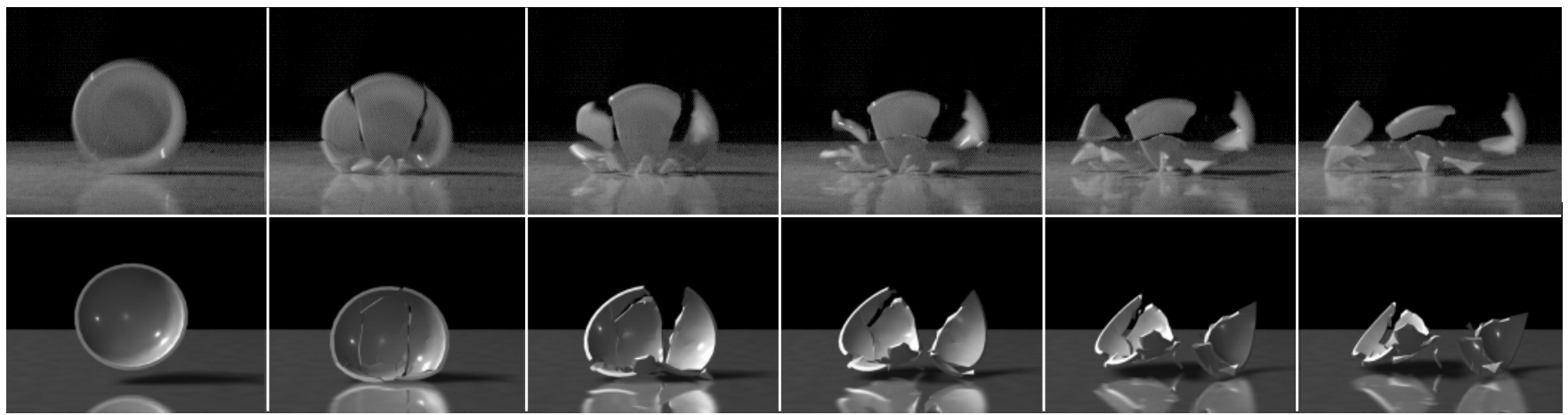

Figure 13: Comparison of a real-world event and simulation. The top row shows high-speed video images of a physical ceramic bowl dropped from approximately one meter onto a hard surface. The bottom row is the output from a simulation where we attempted to match the initial conditions of the physical bowl. Video images are $8 \mathrm{~ms}$ apart. Simulation images are $13 \mathrm{~ms}$ apart.

\section{References}

[1] T. L. Anderson. Fracture Mechanics: Fundamentals and Applications. CRC Press, Boca Raton, second edition, 1995.

[2] D. Baraff and A. Witkin. Large steps in cloth simulation. In SIGGRAPH 98 Conference Proceedings, Annual Confer-

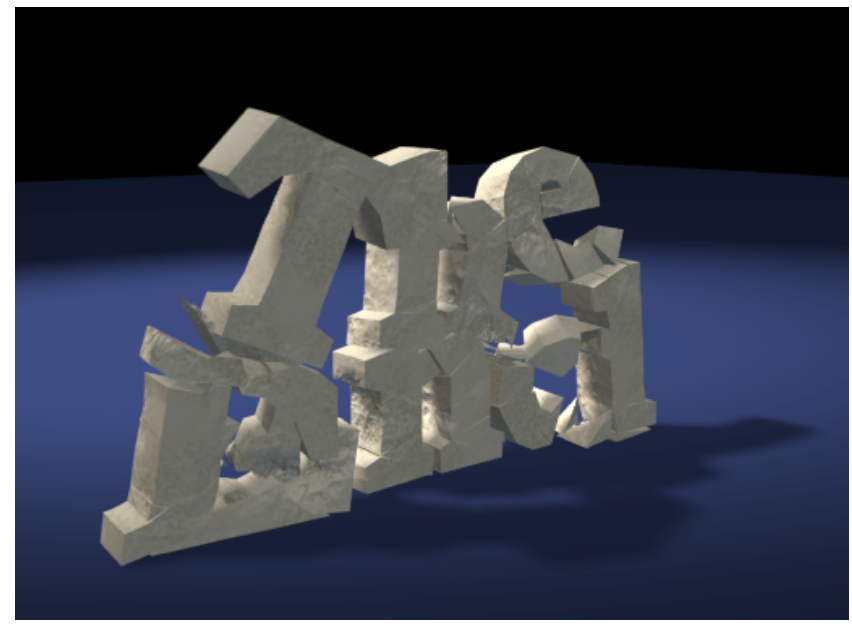

Figure 14: Several breakable objects that were dropped from a height. ence Series, pages 43-54. ACM SIGGRAPH, Addison Wesley, July 1998

[3] R. D. Cook, D. S. Malkus, and M. E. Plesha. Concepts and Applications of Finite Element Analysis. John Wiley \& Sons, New York, third edition, 1989.

[4] T. DeRose, M. Kass, and T. Truong. Subdivision surfaces in character animation. In SIGGRAPH 98 Conference Proceedings, Annual Conference Series, pages 85-94. ACM SIGGRAPH, Addison Wesley, July 1998.

[5] N. Foster and D. Metaxas. Realistic animation of liquids. In Graphics Interface '96, pages 204-212, May 1996.

[6] Y. C. Fung. Foundations of Solid Mechanics. Prentice-Hall, Englewood Cliffs, N.J., 1965.

[7] Y. C. Fung. A First Course in Continuum Mechanics. Prentice-Hall, Englewood Cliffs, N.J., 1969.

[8] K. Hirota, Y. Tanoue, and T. Kaneko. Generation of crack patterns with a physical model. The Visual Computer, 14:126137, 1998.

[9] O. Mazarak, C. Martins, and J. Amanatides. Animating exploding objects. In Graphics Interface '99, June 1999.

[10] M. Neff and E. Fiume. A visual model for blast waves and fracture. In Graphics Interface '99, June 1999.

[11] T. Nishioka. Computational dynamic fracture mechanics. International Journal of Fracture, 86:127-159, 1997. 
[12] A. Norton, G. Turk, B. Bacon, J. Gerth, and P. Sweeney. Animation of fracture by physical modeling. The Visual Computer, 7:210-217, 1991.

[13] W. H. Press, B. P. Flannery, S. A. Teukolsky, and W. T. Vetterling. Numerical Recipes in C. Cambridge University Press, second edition, 1994.

[14] B. Robertson. Antz-piration. Computer Graphics World, 21(10), 1998.

[15] B. Robertson. Meet Geri: The new face of animation. Computer Graphics World, 21(2), 1998.

[16] J. Schöberl. NETGEN - An advancing front 2D/3D-mesh generator based on abstract rules. Computing and Visualization in Science, 1:41-52, 1997.

[17] D. Terzopoulos. Regularization of inverse visual problems involving discontinuities. IEEE Transactions on Pattern Analysis and Machine Intelligence, 8(4):413-424, July 1986.

[18] D. Terzopoulos and K. Fleischer. Deformable models. The Visual Computer, 4:306-331, 1988.

[19] D. Terzopoulos and K. Fleischer. Modeling inelastic deformation: Viscoelasticity, plasticity, fracture. In Computer Graphics (SIGGRAPH '88 Proceedings), volume 22, pages 269278, August 1988. 